\title{
Gastro-oesophageal reflux and idiopathic pulmonary fibrosis: the heart burn in patients with IPF can no longer be silent
}

\author{
Michael Kreuter ${ }^{1}$ and Ganesh Raghu ${ }^{2}$ \\ Affiliations: ${ }^{1}$ Center for interstitial and rare lung diseases, Thoraxklinik, University of Heidelberg, Heidelberg, \\ Germany and German Center for Lung Research (DZL). ${ }^{2}$ Center for Interstitial Lung Diseases, University of \\ Washington, Seattle, WA, USA.
}

Correspondence: Michael Kreuter, Center for interstitial and rare lung diseases, Thoraxklinik, University of Heidelberg, and German Center for Lung Research (DZL), Röntgenstr. 1, 69126 Heidelberg, Germany. E-mail: kreuterauni-heidelberg.de

@ERSpublications

A prospective, randomised trial is needed to determine the safety and efficacy of anti-acid drugs/ proton pump inhibitors in patients with IPF http://ow.ly/A63330ktoGm

Cite this article as: Kreuter M, Raghu G. Gastro-oesophageal reflux and idiopathic pulmonary fibrosis: the heart burn in patients with IPF can no longer be silent. Eur Respir J 2018; 51: 1800921 [https://doi.org/ 10.1183/13993003.00921-2018].

Idiopathic pulmonary fibrosis (IPF) is a progressive interstitial lung disease of complex cause affecting an elderly population where multimorbidity is frequent. There is an increasing understanding of the distinct comorbidities over-represented in patients with IPF and associated with a clinically important impact on symptoms and outcomes [1-4]. More than 40 years ago, MaYs et al. [5] were the first to describe a potential association between gastro-oesophageal reflux (GOR) and IPF. Since then multiple reports have established that gastro-oesophageal reflux disease (GORD) is one of the most prevalent comorbid conditions in IPF. Based on the increased prevalence, it has been hypothesised that GOR-associated microaspirations may have a pathogenic role in IPF. This stimulated further research, including in animal models, which suggested that aspiration of acid refluxate causes the parenchymal damage associated with pneumonitis and increased epithelial permeability with the consequence of stimulated fibrotic proliferation and perhaps of acute exacerbations (summarised in [6]). Therefore, an early dream was that supressing microaspiration may alter the course of IPF, namely by allowing greater symptom management, disease stabilisation and perhaps improved survival [7].

Currently, modalities to decrease risks of microaspiration include: 1) lifestyle modifications; 2) treatment intervention for abnormal acid GOR with anti-acid drugs (AAT); and 3) surgical treatment with laparoscopic anti-reflux surgery to abort the GOR altogether. The latter addresses acid and non-acid GOR, and is currently being investigated in the prospective WRAP-IPF trial (ClinicalTrials.gov identifier: NCT01982968) after initial promising retrospective analyses reported disease stabilisation after laparoscopic fundoplication in patients with IPF $[8,9]$. The situation regarding the use of AAT, i.e. proton pump inhibitors and/or H2-blockers, is more complex. The first large retrospective analysis described that the use of these medications by patients with IPF was associated with a smaller amount of fibrosis on high-resolution computed tomography and was an independent predictor of longer survival [9]. A prospectively designed, pre-specified analysis of the placebo arms of the IPFnet trials using well designed case-report forms to proactively capture data regarding GORD and therapeutic intervention, revealed that the use of AAT for any reason was associated with fewer exacerbations and significantly less worsening of 
forced vital capacity than in patients without AAT during the study period, but without effects on all-cause mortality [10]. However, post hoc and exploratory analyses from the two groups from three clinical trials on pirfenidone in IPF did not reveal differences in the rate of decrease of lung function or mortality when comparing AAT users versus non-users at baseline [11, 12]. Several questions regarding the association of GORD and IPF and management of GORD in patients with IPF need to be addressed in much needed prospective studies [6].

In this context, the report by TRAN and SUISSA [13] in this issue of the European Respiratory Journal opens new insights into critical appraisal of the current evidence in this field. They assessed whether reported effects on survival following AAT may be biased by immortal time bias. This complex phenomenon can arise in the case of misclassification, exclusion or non-accountancy of the period between entry into a cohort and date of first drug exposure, during which the event of interest has not occurred. This can lead to a false reduction in the rate of event occurrence, leading to the misconception that a treatment has protective effects, although no real treatment effects may exist. TRAN and SuISSA [13] identified 10 observational studies and described that four out of the five studies with reported beneficial effects of AAT use on mortality were influenced by immortal time bias, while the five studies that avoided this effect did not observe protective mortality effects of AAT. The need for further studies with proper design is evident, both to assess the effectiveness of AATs in IPF in a real life setting and to explore the potential of beneficial influences of AAT on survival.

The report by TRAN and SUISSA [13] also reveals the need to interpret published reports in general, and those regarding the AAT for IPF in particular, with caution. It alerts the readership and clinicians to be cautious about drawing conclusions from post hoc, subgroup and/or exploratory analyses that merely generate a hypothesis [14]. Comparing pooled hazard ratios from studies affected by immortal time bias (0.46, 95\% CI 0.30-0.69) with those which avoided this effect $(0.94 ; 95 \%$ CI $0.76-1.17)$ one may assume that the direction of this effect could still tend towards a protective effect. While this may, in part, be explained by association with improved survival in patients with IPF allegedly suffering from GORD regardless of treatment $[1,9]$, the retrospective studies do not provide the much-needed answer to the question about the apparent beneficial or adverse effects of AAT [11, 12]. Several confounding factors in the observational studies that introduce risk of bias (e.g. patient populations, diagnosis of GOR, indications for AAT, conservative measures to decrease GOR, concomitant medications, and other comorbidities such as obesity, age, obstructive sleep apnoea or tobacco smoking), and that are known to provoke GOR, need to be taken into consideration when assessing the potential beneficial and harmful effects of AAT in patients with IPF. While the report by TRAN and SuISSA [13] focused on mortality, other important outcomes that are clinically meaningful to patients, such as respiratory hospitalisations, adjudicated acute exacerbations or functional decline, need to be determined in well-defined prospective studies. Such trials need also to assess potential risks in this specific patient population, especially the potential concern of pneumonia and respiratory infections with pre-specified criteria (including theoretical effects of changes to the gut and thus lung microbiome). Furthermore, a potential effect of AAT on the on the absorption, bioavailability and efficacy of concomitant antifibrotic therapies must be studied [6].

In essence, the need to critically appraise evidence accumulated with retrospective, post hoc and/or exploratory analyses is evident. We need to remind ourselves that analyses from such studies are important as they generate hypotheses that warrant further testing in well-designed prospective studies. Indeed, only a well-designed randomised clinical trial can definitely answer the question of the safety and efficacy of anti-acid therapy as a potential treatment in patients with IPF. While the need for such robust evidence may take some time to materialise, patients and clinicians managing patients confronted with this devastating disease must weigh up the potential benefits and risks associated with AAT for IPF on an individual basis. While several clinical trials over the past 25 years have enhanced our current understanding of the clinical course of patients with IPF, we do not have treatment interventions that have aborted the disease process in these patients [15]. Despite the evolved standard of care with the use the antifibrotic drugs (pirfenidone and nintedanib) known to slow the rate of disease progression, these are not readily available to all patients worldwide. Indeed, it is tempting to consider every potential therapeutic option possible that may abort disease progression and/or improve outcomes. While the evidence-based guidelines for treatment of IPF provided a conditional recommendation for treatment with AAT for IPF, it must be reiterated that this was based on low-quality evidence. The pivotal study needed to provide the high-quality evidence can only be accomplished by investigators and sponsors invested to pursue the truth in the interest of enhancing knowledge about the management of patients with IPF. It is about time to heed the appeal from Tran and SuISSA [13] to get on with it. Only a prospective, randomised trial can determine the safety and efficacy of AAT/proton pump inhibitors in patients with IPF and settle the heart burn that has been silent for too long! We are confident that the proven longstanding track record of the concerted efforts of the IPF community-at-large will achieve this soon. 
Conflict of interest: M. Kreuter reports personal fees from GSK and Galapagos, and grants and personal fees from Boehringer and Roche, outside the submitted work.

\section{References}

1 Kreuter M, Ehlers-Tenenbaum S, Palmowski K, et al. Impact of comorbidities on mortality in patients with idiopathic pulmonary fibrosis. PLoS One 2016; 11: e0151425.

2 Raghu G, Amatto VC, Behr J, et al. Comorbidities in idiopathic pulmonary fibrosis patients: a systematic literature review. Eur Respir J 2015; 46: 1113-1130.

3 Raghu G, Rochwerg B, Zhang Y, et al. An official ATS/ERS/JRS/ALAT Clinical Practice Guideline: treatment of idiopathic pulmonary fibrosis. An Update of the 2011 Clinical Practice Guideline. Am J Respir Crit Care Med 2015; 192: e3-e19.

4 Schwarzkopf L, Witt S, Waelscher J, et al. Associations between comorbidities, their treatment and survival in patients with interstitial lung diseases - a claims data analysis. Respir Res 2018; 19: 73.

5 Mays EE, Dubois JJ, Hamilton GB. Pulmonary fibrosis associated with tracheobronchial aspiration. A study of the frequency of hiatal hernia and gastroesophageal reflux in interstitial pulmonary fibrosis of obscure etiology. Chest 1976; 69: 512-515.

6 Johannson KA, Strâmbu I, Ravaglia C, et al. Antacid therapy in idiopathic pulmonary fibrosis: more questions than answers? Lancet Respir Med 2017; 5: 591-598.

7 Raghu G, Yang ST, Spada C, et al. Sole treatment of acid gastroesophageal reflux in idiopathic pulmonary fibrosis: a case series. Chest 2006; 129: 794-800.

8 Raghu G, Morrow E, Collins BF, et al. Laparoscopic anti-reflux surgery for idiopathic pulmonary fibrosis at a single centre. Eur Respir J 2016; 48: 826-832.

9 Lee JS, Ryu JH, Elicker BM, et al. Gastroesophageal reflux therapy is associated with longer survival in patients with idiopathic pulmonary fibrosis. Am J Respir Crit Care Med 2011; 184: 1390-1394.

10 Lee JS, Collard HR, Anstrom KJ, et al. Anti-acid treatment and disease progression in idiopathic pulmonary fibrosis: an analysis of data from three randomised controlled trials. Lancet Respir Med 2013; 1: 369-376.

11 Kreuter M, Wuyts W, Renzoni E, et al. Antacid therapy and disease outcomes in idiopathic pulmonary fibrosis: a pooled analysis. Lancet Respir Med 2016; 4: 381-389.

12 Kreuter M, Spagnolo P, Wuyts W, et al. Antiacid therapy and disease progression in patients with idiopathic pulmonary fibrosis who received pirfenidone. Respiration 2017; 93: 415-423.

13 Tran T, Suissa S. The effect of anti-acid therapy on survival in idiopathic pulmonary fibrosis: a methodological review of observational studies. Eur Respir J 2018; 51: 1800376.

14 Johnson WC, Raghu G. Clinical trials in idiopathic pulmonary fibrosis: a word of caution concerning choice of outcome measures. Eur Respir J 2005; 26: 755-758.

15 Raghu G. Idiopathic pulmonary fibrosis: lessons from clinical trials over the past 25 years. Eur Respir J 2017; 50: 1701209 . 